\title{
La cultura escolar de la Segunda República española. Legislación, teoría y praxis escolar
}

\section{School culture within the Spanish Second Republic. Legislation and pedagogical theory and practice}

\author{
Bienvenido Martín Fraile*1 \\ bmf@usal.es \\ Isabel Ramos Ruiz* \\ mirr@usal.es \\ Pablo Álvarez Domínguez** \\ pabloalvarez@us.es \\ * Universidad de Salamanca, España \\ **Universidad de Sevilla, España
}

\section{Resumen:}

El artículo indaga la presencia de la norma y teoría pedagógicas en la praxis escolar de la escuela primaria durante la Segunda República Española (1931-1936) a través del análisis, mediante el método históricopedagógico, de distintas fuentes documentales: memorias de prácticas de los alumnos de magisterio, libros de actas de escuelas primaria, circulares legislativas y cuadernos escolares. El resultado contempla la aplicación directa de la norma y la teoría educativa en la praxis, evidenciando la acción conjunta de estos ámbitos en la escuela. Muestra el grado de implicación de gran parte del magisterio en los fines e ideales del gobierno republicano, junto a la utilización de la escuela por éste como plataforma para conseguir dichos fines e ideales ${ }^{2}$.

\begin{abstract}
:
This study examines the presence of pedagogical norms and pedagogical theory in primary school practice during the time of the Second Republic through the analysis, according to the historical-pedagogical method, of manifold documentary sources: Teaching practice journals of teacher trainees, primary school minute books, legislative notices and school notebooks. Our results show the systematic and direct implementation of the norms and the pedagogical theory in schools, highlighting the joint action of both spheres in daily school practice. This evidences the degree of involvement of a significant number of teachers with the aims and principles of the Republican government, together with the fact that the government itself used
\end{abstract}

1 Dirección para correspondencia (correspondence address):

Bienvenido Martín Fraile. Departamento de Teoría e Historia de la Educación. Universidad de Salamanca. Escuela Universitaria de Magisterio. Campus Viriato. Avda. Cardenal Cisneros, s/n. 49029 Zamora (España).

2 Este artículo se enmarca dentro del proyecto de investigación financiado por el Ministerio de Economía y Competitividad del Gobierno de España, proyecto número EDU2013-44129-P. 
La cultura escolar de la Segunda República española. Legislación, teoría y praxis escolar

Bienvenido Martín Fraile, Isabel Ramos Ruiz y Pablo Álvarez Domínguez

\section{Palabras clave:}

Cultura empírica; cultura escolar; escuela primaria; etnografía escolar. schools as a platform to achieve their aims and ideals.

\section{Key words:}

Empirical Culture; School Culture; Primary School; School Ethnography.

\section{Résumé:}

L'article explore la présence de la norme et de la théorie pédagogiques dans la pratique scolaire de l'école primaire pendant la Seconde République Espagnole (1931-1936) à travers I'analyse, en utilisant la méthode historico-pédagogique, de différentes sources documentaires : mémoires des stages (pratiques) des étudiants de l'École Universitaire de Formation de Maîtres, livres des actes des écoles de primaire, circulaires législatives et cahiers scolaires. Le résultat envisage l'application directe de la norme et de la théorie éducative dans la pratique, et met en évidence l'action commune de ces domaines à l'école. Cela montre le degré d'implication d'une grande partie du professorat dans les objectifs et les idéaux du gouvernement républicain, et l'utilisation de l'école par celui-ci comme tremplin pour obtenir ces objectifs et idéaux.

\section{Mots clés :}

Culture empirique; culture scolaire; école primaire; ethnographie scolaire.

Fecha de recepción: 31-5-2017

Fecha de aceptación: 11-9-2018

\section{Introducción}

El conocimiento de la escuela y la cultura generada por ésta se ha convertido en un objetivo de la historia de la educación en las últimas décadas. Los historiadores quieren conocer "las prácticas y discursos" (Escolano, 2006, p. 23) Ilevados a cabo en el interior del aula donde maestros y alumnos han permanecido mucho tiempo actuando en silencio, sin que su trabajo viese la luz y alguien se diese cuenta de la importancia que tiene para desentrañar la realidad escolar e interpretar el modelo educativo.

Al mismo tiempo, esta nueva línea de investigación no ha merecido la atención de los historiadores hasta hace unos años. En la actualidad el interés por la microhistoria escolar y la intrahistoria de la educación se hace visible tras el análisis que proporcionan los ámbitos que influyen en la cultura escolar: práctico, discursivo y normativo, también denominados empírico, teórico y político. Una perspectiva micro que se sirve de variados testimonios como son las fuentes materiales y orales, además de las escritas e iconográficas (Escolano, 2007, p. 15). 
Distintos estudios realizados en los últimos años acerca de la cultura escolar recogen las relaciones existentes entre la praxis curricular y la legislación. Un ejemplo es el trabajo de las profesoras de la Universidad Complutense y de la de Alcalá, Rabazas y del Pozo (2013) donde analizan, a través de memorias escolares, la influencia de la política educativa en la realidad escolar del primer franquismo. Según las autoras, la historia de la escuela se construye a partir de la praxis escolar que desarrollan los maestros. Praxis determinada por la mentalidad y la dinámica profesional de los maestros más que por los cambios políticos que se producen.

De igual manera, Barceló, Comas y Sureda (2016) utilizan las Memorias de Prácticas de los alumnos de Magisterio con el objetivo de descubrir el pasado escolar, el día a día en las aulas, también denominado la caja negra de la escuela. Los autores afirman que el conocimiento del período histórico estudiado, -la escuela franquista de la década de los años cuarenta-, necesita al margen de la norma ideológica, otros aspectos referidos a la cotidianeidad del aula que demuestran la existencia de continuidades que se resistieron a la voluntad política de imponer cambios, afirmando la separación evidente de lo legislado y la praxis real del aula.

Los cuadernos de los alumnos también han sido objeto de una investigación creciente para descubrir la vida diaria dentro del aula, estudiando tanto el currículo, las materias y la metodología contenidos en sus páginas, como los sentimientos de los niños y la educción emocional que reciben a través de sus escrituras. Desde esta perspectiva se remarcan los trabajos de Martín y Ramos $(2014,2015)$ y Beas y González (2016).

\section{Marco empírico}

El trabajo analiza los tres ámbitos influyentes en la cultura escolar a partir de fuentes documentales: el legislativo lo proporciona el estudio pormenorizado de las circulares y decretos publicados durante el primer bienio del periodo republicano y, en un segundo momento, tras el triunfo en las elecciones del Frente Popular en febrero de 1936; la teoría educativa se presenta por medio de los testimonios recogidos en los cuadernos de Memorias de Prácticas de alumnos/as de Magisterio que informan de los principios y teorías de la formación normalista recibida; y la praxis curricular se muestra en dichas Memorias, en la Actas de Juntas 
de maestros, en los cuadernos escolares individuales y colectivos de los alumnos, y en los de preparación de lecciones del maestro. El objetivo final del estudio es mostrar y recoger si realmente llegó a producirse la aplicación en el aula (praxis escolar) de las prescripciones legislativas y teóricas que ofrecían las disposiciones legales, o si por el contrario, se produjo una desunión de los tres ámbitos o no llegó a llevarse a cabo plenamente su incorporación a la vida escolar.

El método de investigación utilizado en la investigación ha sido el histórico-pedagógico. La historia de la educación escolar se engloba dentro de una historia general política y social que no puede desligarse de la historia general. Por otro lado, la interpretación es pedagógica al tratar sobre una realidad e institución educativa consolidada a lo largo del siglo XIX y que alfabetiza y proporciona los rudimentos de cultura a la población infantil. El tema de análisis se centra en el contexto histórico y pedagógico del régimen político de la Segunda República. A la euforia republicana inicial le siguió una larga temporada de olvido impuesto durante la etapa de la dictadura franquista (1936-1975). Luego vinieron unos años de emoción contenida por la vuelta a escena de aquellos principios e ideales educativos con la llegada de la democracia desde 1975. La democracia no consiguió totalmente una revisión del esfuerzo de la Segunda República, pero ahora, cercano el centenario, se inicia el momento de ir descubriendo realmente qué se consiguió. La mirada se dirige hacia unos años en que las dos terceras partes de la población pertenecían al ámbito rural, muy atrasado, y sólo una tercera parte tenía acceso a los beneficios que reportaba la vida en núcleos urbanos. Este es el marco teórico que impulsa a investigar la práctica escolar de esta época y comprobar con datos y hechos históricos los logros y fracasos cosechados.

Diversos estudios han puesto de relieve el valor de las fuentes documentales inéditas circunscritas al ámbito de la cultura escolar escrita (Viñao, 1996), que constituyen, junto con los libros y manuales escolares un elemento que enriquece y complementa la historia escolar En el trabajo realizado se han consultado fundamentalmente los cuadernos de las Memorias de Prácticas de alumnos de Magisterio, los cuadernos oficiales de la escuela como los Libros de Actas de Juntas y los cuadernos de alumnos, tanto individuales de deberes como colectivos de rotación y también en menor cuantía los de preparación de lecciones de los maestros.

Todos ellos han sido sometidos a crítica histórica para determinar su 
validez formal y de contenido. Se han analizado cincuenta cuadernos de la etapa republicana, de varones y mujeres, unitarias principalmente y algunas graduadas, del Fondo Documental de cuadernos del CeMuPE (Centro Museo Pedagógico de la Universidad de Salamanca). Pertenecen a un contexto geográfico concreto, las provincias de Orense, Lugo, Zamora, Valladolid, Salamanca y Cáceres, en el oeste peninsular que limita con Portugal o cercano al mismo.

El estudio pone de relieve el valor de estas fuentes documentales inéditas, que vienen a ser el testimonio directo y personal de lo que sus protagonistas consideraban que era lo más importante dentro de las aulas. Son los corresponsales que nos introducen con sus crónicas en la escuela de una época ya pasada, de la cual ha quedado la huella grabada en las escrituras. En palabras de B. Sustar (2011) "los testimonios escritos permiten observar sus sentimientos, expectativas y juicios de una manera tal que no se encuentran expresados en los documentos formales de la historia del sistema educativo".

Es hora, después de casi cien años, de recoger la huella escrita dejada en los cuadernos y reinterpretar la historia escolar analizando la información que proporcionan dichas fuentes y llevar a cabo su interpretación, contrastándola con la información proporcionada por la legislación educativa o la teoría pedagógica.

\section{Resultados y discusión}

\section{La cultura de la escuela}

En las últimas décadas la historiografía educativa viene trabajando en un nuevo campo de estudio e investigación, la cultura de la escuela, presentándose como el conjunto de normas (saberes y conductas) y prácticas que a lo largo de los siglos la escuela ha generado. Así la define Dominique Juliá:

un conjunto de normas que definen saberes a enseñar y conductas a inculcar y un conjunto de prácticas que permiten la transmisión de estos saberes y la incorporación de estos comportamientos, estando orientadas estas normas y prácticas a finalidades que pueden variar según las épocas (finalidades religiosas, sociopolíticas o simplemente de socialización). (Juliá, 1995, p. 354) 
El profesor Escolano (2006) introduce un tercer elemento constituyente en la cultura escolar, el teórico o discursivo. Son, pues, tres los ámbitos que influyen en la cultura escolar: práctico, discursivo y normativo, también denominados empírico, teórico y político.

Aceptando, pues, tres factores determinantes en la cultura generada por la escuela a lo largo de la historia, se inicia su estudio en relación a la escuela primaria de la Segunda República. Es ahora cuando los deseos de reforma del pueblo español desatendidos por las instancias políticas desde décadas atrás comienzan a hacerse realidad. La escuela, centro de educación y cultura, será uno de los campos prioritarios de actuación de los nuevos gobernantes que veían en el analfabetismo la mayor lacra del país, con la que había que acabar si lo que se quería conseguir era un pueblo culto, base para consolidar la democracia. Para ello, era necesario comenzar por abajo, por la escuela primaria, continuando con el desarrollo de la política de construcciones iniciada en la etapa anterior, aumentando la plantilla de maestros y dotando a la escuela de recursos. Todo un plan bien pensado, detallado y minucioso, resumido en la expresión popular "más escuelas y mejores maestros", lo que requería una puesta en práctica rápida y ágil. No obstante, hay que señalar que la Segunda República lo que hace es ratificar y dar cobertura institucional al proyecto iniciado tiempo atrás por la Ilustración Libre de Enseñanza (ILE), y del que ya se han hecho eco muchos maestros progresistas en sus escuelas.

También hay que señalar que aunque la Constitución republicana de 1931 tendrá muy poca vigencia, sentará las bases de un sistema educativo encaminado a la consecución de un país desarrollado y democrático. Este periodo llevó al pueblo español la ilusión hacia un nuevo régimen político que solucionase los graves problemas que el país arrastraba desde siglos atrás y que no permitían un desarrollo tal y como el resto de los países europeos habían alcanzado. La democracia implantada estimuló la cultura, algo de lo que el pueblo llano no participaba, despertando verdadero entusiasmo y convirtiéndose en la principal acción del gobierno que la proyecta hacia la escuela con la intención de mejorarla.

\section{Legislación educativa y praxis escolar}

Con anterioridad a la Constitución de 1931 se aprueban una serie de decretos que cambiarán la concepción de la enseñanza y mostrarán la política educativa republicana encaminada hacia la "escuela única", 
responsabilidad solo del Estado, sin la presencia de la Iglesia ${ }^{3}$. Por su parte la Constitución en el artículo 48 establece las bases de la educación: "El servicio de la cultura es atribución esencial del Estado, y lo prestará mediante instituciones educativas enlazadas por el sistema de la escuela unificada". La enseñanza primaria será gratuita y obligatoria; se reconoce y garantiza la libertad de cátedra; la enseñanza será laica y hará del trabajo el eje de su actividad metodológica, inspirándose en ideas de solidaridad humana. No obstante, se reconoce a las Iglesias el derecho, sujeto a inspección del Estado, de enseñar sus respectivas doctrinas en sus propios establecimientos (art. 26).

La lucha por conseguir la escuela única alcanza a temas como la libertad de expresión, la libertad religiosa y el respeto a la conciencia del niño y del maestro. Según Capitán Díaz "la libertad religiosa era, para el Gobierno Provisional de la República, la clave que situaría a España al nivel "moral y civil" de las democracias europeas" (Capitán, 1986, p. 893).

La escuela se va a convertir en "el arma ideológica de la revolución española", en frase del propio Director General de Primera Enseñanza Rodolfo Llopis (1927). Las medidas que se adoptan son variadas, entre las que destacan la organización y promoción de la escuela primaria, desarrollo de la política de construcciones escolares, mejor preparación de los maestros a través de un nuevo plan de estudios y el aumento en su número, el fomento de un modelo educativo que responde a los principios de la Escuela Nueva o creación de instituciones educativas complementarias. Así, en los primeros tiempos de la proclamación del nuevo estado existe una euforia política que impulsa las reformas demandadas desde hace mucho tiempo atrás y que afectan a la educación. Las primeras decisiones adoptadas intentan, así, arbitrar medidas de urgencia para transmitir el modelo educativo propio a implantar.

¿Hasta dónde llegará la República, que ha comprendido y profundizado nuestras necesidades escolares y que en numerosos Decretos ha hecho pública su intención de elevar nuestro medio social con instituciones complementarias de la Escuela, tales como el patronato de Misiones pedagógicas, encargado de difundir en España las obras benéfico-educativas? ${ }^{4}$

3 Decreto de 6 de mayo de 1931, por el que la instrucción religiosa no será obligatoria en las escuelas primarias ni en ninguno de los demás centros dependientes del Ministerio de Instrucción Pública y Bellas Artes. Colección Legislativa de España, T. CXXIV. pp. 42-43

4 CEMUPE. FDoc. (128) Caja 2. Alumno José Guerra Cid. Cuaderno de Memorias de 
La cultura escolar de la Segunda República española. Legislación, teoría y praxis escolar

Bienvenido Martín Fraile, Isabel Ramos Ruiz y Pablo Álvarez Domínguez

En los dos periodos que el gobierno de la República estuvo en manos de la izquierda, el poder político diseñó un programa con los planteamientos teóricos educativos que desde hacía décadas defendía la ILE. Escuela unificada, gratuidad y obligatoriedad de la escuela primaria, reconocimiento de la libertad de cátedra, enseñanza laica, coeducación, serán algunas de las ideas pedagógicas que quedaron plasmadas en la Constitución de 1931 en su artículo 48.

El número de 40 alumnos es hasta cierto punto tolerable, más bien excesivo para las tareas escolares, que para ser eficaces, la moderna pedagogía indica 30 para cada grado, asesorado con el prestigioso consejo de los inimitables maestros D. Francisco Giner de los Ríos y D. Rufino Blanco, ambos propulsores infatigables del sistema de graduación y a cuyas fuentes deben acudir los maestros presentes y futuros, no ya de España sino del mundo, junto con el gran Pestalozzi. ${ }^{5}$

Una vez perfilado el programa fue necesario ponerlo en marcha y planificar distintas actuaciones desde diversas instancias. La Inspección Educativa será uno de los ejes activos a través de la cual las ideas pedagógicas llegarán a las escuelas primarias donde los maestros las escucharán directamente de los inspectores en las reuniones programadas desde los Centros de Colaboración Pedagógica ${ }^{6}$. Tal y como señala Antonio Molero (2010, pp. 170-179) en estas sesiones entre 1932 a 1934 se tratan temas sobre diversos aspectos, siendo uno de ellos el relacionado con los principios constitucionales.

La Revista de Pedagogía se convierte también en un vehículo transmisor en estos momentos. Constituye un medio de difusión hacia el profesorado primario que traslada continuamente informaciones al magisterio para que se sume y adhiera a las nuevas medidas que plantea la política del gobierno republicano. Por un lado, hace visibles el conjunto de motivos que este colectivo viene arrastrando por la dejadez y desidia del gobierno monárquico de Alfonso XIII: el menosprecio a la educación y la cultura, la ausencia de medidas que corrijan la falta de alimentación y

Prácticas de Magisterio (CMPM). Escuela unitaria de párvulos de Pueblica de Valverde (Zamora). 1936 (mayo).

5 CEMUPE. FDoc. (178) Caja 2. Alumno Julián Méndez Becedas. (CMPM). Escuela aneja a la Normal de maestros de Salamanca. 1938.

6 CEMUPE. FDoc. (26) Libro de Actas de la Junta de Maestras de la escuela graduada de niñas de Cabezuela del Valle (Cáceres). 1932. Acta nº 17. 
vestido de la infancia, la lacra que supone el déficit de plazas escolares para tantos niños que reclaman un puesto escolar ${ }^{7}$, el perjuicio que, tanto a al magisterio como a los niños provoca la inexistencia de condiciones pedagógicas e higiénicas en las escuelas y el trato vejatorio sufrido por este colectivo por los sueldos míseros que ha percibido, una muestra más del olvido al que la monarquía le ha sometido durante tanto tiempo. De otro lado, y una vez presentadas las privaciones y condiciones de vida profesional y personal de los maestros, se les ilusiona con la nueva etapa que comienza para la cultura y la vida en el país, objetivos prioritarios para los nuevos gobernantes republicanos.

Para conseguir el entusiasmo del magisterio y la ayuda a las nuevas propuestas se exponen varias medidas: Una, mejorando el trabajo en el aula diariamente, aplicando nuevas técnicas y procedimientos pedagógicos que consigan elevar el nivel cultural y educativo de los alumnos; y dos, inculcando en los niños los ideales republicanos, un nuevo espíritu que busca la transformación del pueblo español a través del sistema político de la democracia, que trae la libertad, la solidaridad y la civilidad. Son los valores a impulsar en la escuela por el maestro que, en definitiva, representan los principios de la nueva escuela o escuela nueva que se quiere implantar y que impulsan la formación de un nuevo hombre, un nuevo humanismo, el de ciudadano participativo, respetuoso y responsable.

La traducción a la praxis escolar de estas recomendaciones se puede apreciar en un rastreo y análisis de los cuadernos referidos a este período histórico: escrituras de niños que reflejan el uso de metodologías y principios de la Escuela Nueva, como Decroly ${ }^{9}$ o Froebel; o testimonios de maestros/as en los Libros de Actas de Juntas Locales de Instrucción Primaria y Juntas de Maestras.

Otro recurso utilizado por el Ministerio de Instrucción Pública y BeIlas Artes serán las circulare ${ }^{10}$ que llegan directamente a las aulas, expo-

7 CEMUPE. FDoc. (27). Libro de Actas de la Junta Local de Instrucción Primaria de Cabezuela del Valle (Cáceres). 1928. Recoge la reunión con la inspectora de zona sobre las necesidades de puestos escolares.

8 Notas del mes. "La función pedagógica del magisterio" Revista de pedagogía,115, julio- 1931. También en esta misma sección, diciembre de 1931, p. 567.

9 CEMUPE. FDoc. (166-175). Cuaderno de deberes del alumno Ricardo García, donde el maestro realiza una enseñanza basada en el método pedagógico de Decroly a través de los centros de interés. Escuela de Retortillo (Salamanca), 1934-35.

10 Dos son las circulares que utilizamos a lo largo de este trabajo. La primera está firmada por el Director General de Primera Enseñanza, Rodolfo Llopis, el 12 de Enero de 
La cultura escolar de la Segunda República española. Legislación, teoría y praxis escolar

Bienvenido Martín Fraile, Isabel Ramos Ruiz y Pablo Álvarez Domínguez

niendo temas que deben aplicarse al ámbito escolar, y cuya enseñanza se encuentra contenida en los cuadernos de los alumnos.

Una primera circular notifica a todas las escuelas nacionales el envío de ejemplares de la Constitución que las Cortes constituyentes acaban de votar y en donde se resalta el mensaje que los maestros deben transmitir a los alumnos:

los maestros deben aprovechar esta circunstancia para hacer a sus alumnos una serie de lecciones, en las que sea la Constitución el tema central de la actividad escolar. Deben explicar a los niños lo que significa una Constitución para las democracias; (...) y cómo la República actual, al promulgar la Constitución, señala un momento histórico en el proceso de liberación que desde hace años vive dramáticamente el pueblo español. (Circular de 12 de Enero de 1932)

Junto a la recomendación de la enseñanza del significado de la Constitución y del concepto de ciudadanía en la escuela (Martín y Ramos, 2010), se añade la responsabilidad que tienen los maestros en la renovación profunda del país "y de quienes la República espera han de cumplirla con fervoroso entusiasmo" (Circular de 12 de enero de 1932).

Una segunda circular fechada el 28 de marzo de 1936 añade:

La Constitución de la República tiene una serie de artículos que constituyen máximas morales y civiles que pueden y han de ser lecciones permanentes. Una de ellas es el primer párrafo del articulo $1 .{ }^{\circ}$; otra, el artículo $2^{\circ}$; otra, el primer párrafo del artículo 25; otra, el artículo 28; otra, el primer párrafo del artículo 44; otra, el primer párrafo del artículo 46; otra, el primero» cuarto y quinto párrafos del artículo 48. Destacarlos con cuadros en las paredes de las salas de clase, en los trabajos escritos y en los cuadernos de labor para que aparezcan permanentemente ante los ojos de los alumnos, comentarlos con frecuencia, discernir su profundidad ética y contribuir a la formación de la conciencia civil y alcanzar este sentido de la responsabilidad personal y colectiva, que es la más alta conquista a que aspira la República. (Circular de 28 de marzo de 1936).

La referencia explícita de lo indicado por la superioridad, se encuentra en los cuadernos de los alumnos, bien como copia, dictado y otros

1932 y la segunda por el Ministro de Instrucción Pública, Marcelino Domingo, el 28 de Marzo de 1936. 
ejercicios, entre los que destaca por su insistencia y pulcritud el de un alumno de la localidad de San Miguel de la Ribera (Zamora) ${ }^{11}$. Supone un ejemplo notable de la no neutralidad de la escuela, que siempre ha respondido al poder establecido.

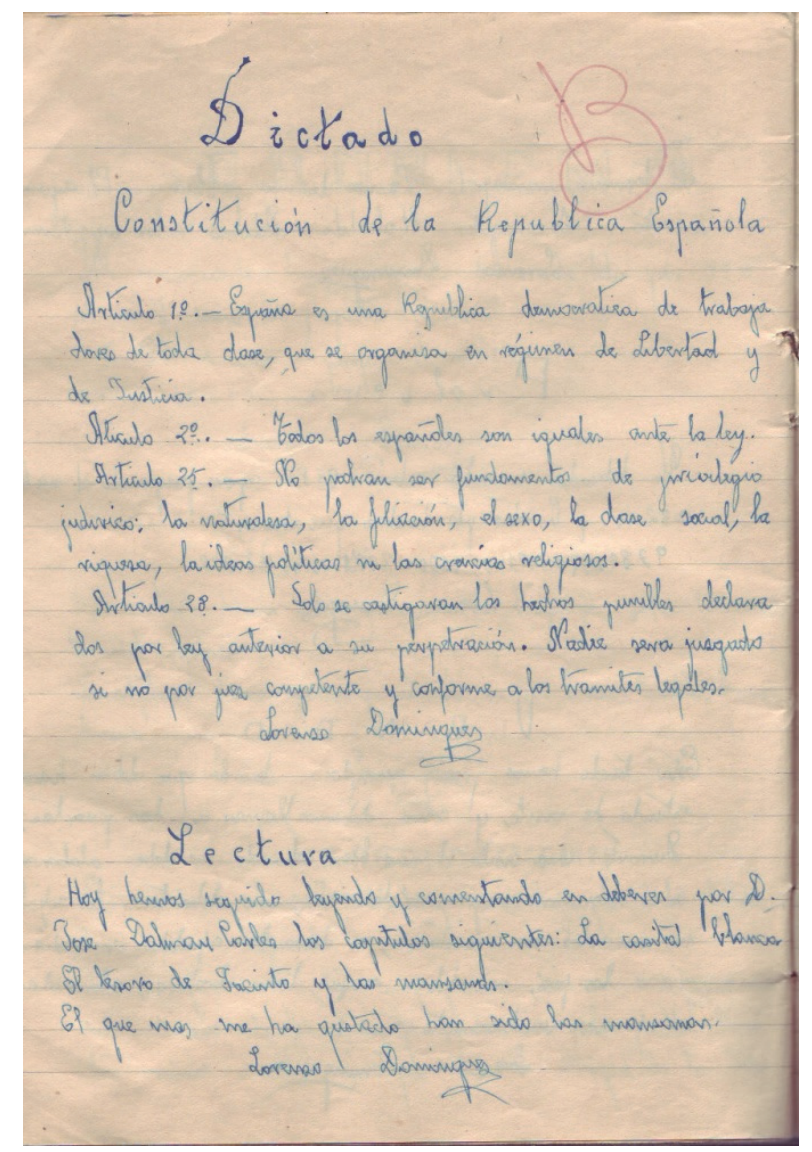

Figura 1. Página de cuaderno con la copia de artículos de la Constitución de 1931

También aparece esta recomendación de la superioridad en los mismos términos que indica la circular en el cuaderno de rotación de la escuela de la Tuda ${ }^{12}$ donde el maestro plantea a los alumnos realizar una

11 CEMUPE. FDoc. (335). Cuaderno de deberes del alumno Lorenzo Domínguez. Escuela nacional de San Miguel de la Ribera (Zamora), 1936.

12 CEMUPE. FDoc. (261). Cuaderno de rotación. Escuela mixta de La Tuda (Zamora). Curso escolar 1934-35. 
La cultura escolar de la Segunda República española. Legislación, teoría y praxis escolar

Bienvenido Martín Fraile, Isabel Ramos Ruiz y Pablo Álvarez Domínguez

actividad de análisis gramatical con la frase "El gobierno de España es republicano".

El análisis de este estudio se centra en otro cometido impulsado por la disposición legal: la escuela ha de ser laica. Así, se dice textualmente que "la escuela, por imperativo del artículo 48 de la Constitución, ha de ser laica. Por tanto, no ostentará signo alguno que implique confesionalidad, quedando igualmente suprimidas del horario y del programa escolares la enseñanza y las prácticas confesionales" (Circular de 12 de enero de 1932).

Existen muestras de la conflictividad que supuso esta normativa en el ejercicio diario de la escuela primaria. Testimonios escritos y tratados en expedientes abiertos desde las Inspecciones de Primera Enseñanza, confirman el no cumplimiento de la citada normativa con rigurosidad. La propuesta se analiza en el reflejo extraído de las memorias de los alumnos de Magisterio tras su paso por las escuelas al realizar sus prácticas. De entrada, en las memorias estudiadas que recogen las prácticas antes de la promulgación de la Circular de 12 de enero de 1932 se aprecia únicamente una diferencia en las prácticas religiosas según se trate de un centro urbano o del ámbito rural. Los alumnos que realizan las prácticas en la ciudad en las escuelas anejas a la Normal de Magisterio no informan nada de esta casuística; en cambio aquellos otros alumnos que realizan las prácticas en escuelas rurales dejan constancia de la enseñanza de la religión en las aulas: "Al Ilegar la hora de salida se rezaban las oraciones de costumbre"; "Por la tarde daba principio la clase lo mismo que por la mañana rezando las oraciones de entrada"13.

Dicho esto, a partir de esta fecha, enero de 1932, no hay ninguna Memoria en la que aparezcan prácticas religiosas en la escuela, hecho que confirma la efectividad de la norma en su aplicación en cuanto a la eliminación de la enseñanza de la religión en la escuela.

Por tanto, se puede asegurar que la introducción de nuevos contenidos en el ámbito escolar como es el estudio de la enseñanza de principios democráticos y de ciudadanía, o la exclusión de otros como los de índole religiosa, se llevan a cabo con éxito en los años de existencia de la Segunda República, respondiendo a las nuevas líneas ideológicas que se plasman en el discurso normativo educativo y que se proyecta hacia la enseñanza primaria.

13 CEMUPE. FDoc. (124). Caja 5. Alumna Leovigilda Nieto Andrés. (CMPM). Escuela nacional de Montamarta (Zamora). 1931. 


\section{Teoría educativa y praxis escolar}

La República conocía la raíz de los denominados males nacionales, el problema pedagógico y el problema político. El plan consistía en aunar ambos en uno retomando la vieja idea de los ilustrados de sumar la revolución política a la educativa. Es el ideal de la revolución social basada en una transformación educativa del país que acabe con el tradicionalismo y que apunte a las nuevas ideas renovadoras para terminar con aquél y permita progresar en la línea que han hecho antes otros países avanzados de Europa. La sociedad debe progresar hasta convertirla en culta y liberadora, sin dogmatismos.

Este planteamiento cultural, propio de la ILE, se verá respaldado por el socialismo y su movimiento obrero, constituyendo una ayuda inestimable que mantiene la tesis de la revolución social auspiciada desde la educación (Barreiro, 1983, pp. 209-215).

Las ideas de la ILE pretendían un hombre nuevo y requerían un cuadro de intelectuales que tras conseguir poder político pusieran en acción los objetivos humanistas que encerraba la nueva pedagogía. Una nueva pedagogía que es necesario arraigar en una nueva escuela donde se pongan en práctica los planteamientos teóricos diseñados. Para ello, y por una parte, los maestros en activo han recibido Cursillos Pedagógicos y han asistido a congresos en donde se les ha mostrado estos principios educativos; y por otra, se incorporan estos contenidos en los programas de formación de los futuros maestros en las Escuelas Normales de Magisterio. De inicio, la República solicita el respaldo de su modelo educativo en todas las instancias relacionadas con la educación que puedan colaborar. Su búsqueda se va a centrar principalmente en el magisterio, en la figura de un maestro capaz de proyectar los principios, ideales, contenidos y metodología a la nueva escuela (Martín, 2015), en el maestro que es educador por encima de instructor (Circular de 12 de enero de 1932), lo que se va a conseguir con vocación, aptitud profesional y una buena formación pedagógica (Plan Profesional de 1931). Tal y como lo señalaba Cossío: "dadme un buen maestro y él improvisará el local de la Escuela si faltase, él inventará el material de enseñanza, él hará que la asistencia sea perfecta" (Cossío, 1929, p. 217).

En las Memorias de Prácticas de los propios alumnos de Magisterio se observa que todos ellos reconocen estas cualidades y la predisposición en el maestro. Baste un ejemplo entre muchos otros: "Claro que no 
La cultura escolar de la Segunda República española. Legislación, teoría y praxis escolar

Bienvenido Martín Fraile, Isabel Ramos Ruiz y Pablo Álvarez Domínguez

en todas las escuelas nacionales se cuenta con medios apropiados para ello, por lo menos hemos visto como (sic) el interés de la Maestra y su vocación para la enseñanza puede suplir mucho la falta de material para llevar a cabo la labor de clase ${ }^{\prime 14}$

En las circulares se combinan normativa y teoría educativa. Uno de los principios educativos que están en la base del movimiento de la Escuela Nueva es vitalizar la escuela, conectarla con la vida, haciendo que ésta penetre dentro de la escuela. Los niños no deben aprender para la propia institución, sino para la vida. Debe evolucionar al mismo ritmo que lo hace la sociedad en la que se encuentra y ayudar a resolver los problemas de la vida cotidiana. Otros principios educativos que impulsa la nueva escuela es el amor al trabajo, el activismo del alumno mediante una metodología basada en centros de interés que responda a la curiosidad del niño y sus interrogantes. Todos estos planteamientos pedagógicos se recogen en las escrituras de maestros y alumnos, mostrando los cambios que se van produciendo en la escuela republicana.

Ejemplos de la metodología que se plantea en el trabajo diario:

En esta escuela se sigue el programa con arreglo al método Decroly, método que tiene la enorme ventaja de enlazar los estudios de las diferentes asignaturas alrededor de un centro de interés de tal manera que, manejado por un buen maestro, constituye un elemento altamente beneficioso para la formación intelectual de los escolares y más aún si el alumno no se limita a observar y escuchar, sino a trabajar, a hacer, explorar, investigar, surgiendo con esta labor así orientada el alumno capaz de desenvolverse por sí mismo, animado por la actividad creadora que espontáneamente surge en el niño cuando la escuela es verdaderamente activa ${ }^{15}$

La enseñanza es cíclica y práctica bajo el principio de instruir deleitando. En base a razonamientos y experiencias: aquello que se hace se aprende mejor que aquello que se ve y se oye. El maestro después de explicar pregunta a los chicos para comprobar si se han entendido los conocimientos. También se apoya de dibujos y experiencias prácticas ${ }^{16}$

14 CEMUPE. FDoc. (170) Caja 5. Alumna Araceli Alonso Sandoval. (CMPM). Escuela graduada aneja a la Normal de Salamanca. 1935.

15 CEMUPE FDoc. (130-131). Caja 2. Alumno Manuel Chinchilla Orantes. (CMPM). Escuela "Blanco de Cela", de Astorga (León). 1932-33.

16 CEMUPE. FDoc. (129). Caja 2. Memoria de prácticas magisterio del alumno Felipe Hernández Garzón. Escuela de Villardondiego (Zamora). 1932. 
La totalidad de las memorias revisadas hablan de la metodología docente en perfecta sintonía con los principios pedagógicos y las teorías educativas de la Escuela Nueva, especialmente de Decroly y Pestalozzi.

Procuraba dar las materias de forma intuitiva, teniendo presente que lo que penetra por los sentidos queda más grabado y la enseñanza es más amena, valiéndome del abundante material que nos brinda la naturaleza. Así se puede comprobar que si a los niños se presenta el objeto natural la enseñanza es completa, si sólo en láminas o el dibujo en el tablero (el gran recurso del maestro) solo se logra la mitad; pero si se enseña en abstracto la enseñanza no vale nada. ${ }^{17}$

En otra memoria se recoge el cometido metodológico intuitivo y experimental:

Más que principios sistemáticos, colocaban a los niños en presencia de los hechos y realidades, enseñándoles el hábito de la observación, de semejanza, etc; partiendo de lo conocido a lo desconocido, de lo particular a lo general, de lo concreto a lo abstracto: Colocando al alumno en actividad y libertad, fomentando la imaginación y el desenvolvimiento de la personalidad del niño. ${ }^{18}$

La pedagogía de la ILE también está presente en las memorias analizadas, como en esta escuela donde no hay libros de texto:

En cuanto a la organización de la enseñanza ésta es objetiva, a base de ejercicios prácticos, de inserción, ilustración y redacción. Una enseñanza intuitiva, de conversación animada y viva para que el alumno reflexione y sienta la sensación del descubrimiento de la investigación como obra del mismo, es decir, acumulando datos por los que los niños por sí mismos llegaban a comprender lo general y lo abstracto. ${ }^{19}$

La última propuesta que se recoge de las circulares hace referencia a la unión que debe establecerse entre la escuela y el pueblo. La escuela

17 CEMUPE. FDoc. (7). Caja 2. Alumno Patricio Nicolau. (CMPM). Escuela unitaria de niños de Rodilana (Valladolid). 1932.

18 CEMUPE FDoc. $(92,98)$. Caja 5. Alumna $M^{a}$ de las Mercedes Crespo Asensio. (CMPM). Escuela nacional graduada de niños aneja a la Normal (Zamora).1932.

19 CEMUPE. FDoc. $(92,98)$. Caja 5. Alumna $M^{a}$ de las Mercedes Crespo Asensio. 
La cultura escolar de la Segunda República española. Legislación, teoría y praxis escolar

Bienvenido Martín Fraile, Isabel Ramos Ruiz y Pablo Álvarez Domínguez

en contacto con la vida real. Es el Ilamamiento a los maestros para realizar salidas escolares, excursiones, paseos y visitas que permitan conocer a los niños el entorno de la escuela al "utilizar los grandes valores educativos que encierra el ambiente geográfico" (Circular de 12 de enero de 1932). Este aspecto también aparece en los testimonios de maestros/as de los Libros de Actas de las Juntas de maestras de escuelas primarias ${ }^{20}$, en cuadernos de escuelas y en las Memorias de Prácticas de Magisterio. Los alumnos de Magisterio relatan en sus memorias lo que sucedía en las aulas y que se confirma con las escrituras de los niños en sus cuadernos.

Un futuro maestro nos explica que "se realizan ejercicios sobre los fenómenos naturales, se llevan diarios de excursiones históricas, agrícolas, de artes, etc. Las lecciones de observación son llevados por los Maestros al trabajo manual, dibujos, trabajos de inserción, juegos, gimnasia, etc" ${ }^{21}$

Se constatan asimismo actividades educativas complementarias:

Ayer como estaba tan buen día nos fuimos de paseo por el camino de las arenas directas al puente la garra vimos por el camino un ombre que estaba esparamando abono pa que diera producto la tierra despues se siembra el trigo cuando va creciendo se...(sic $)^{22}$

Cada semana se dan a las niñas mayores una pequeña excursión o paseo a los alrededores de la población. En este mes hemos realizado en unión de las niñas y profesoras de otras dos escuelas, una excursión al Monasterio de Osera. ${ }^{23}$

20 CEMUPE. FDoc. C. OFICIALES. ACTAS (26) Libro de Actas de Junta de Maestras de la escuela graduada de Cabezuela del Valle (Cáceres). 1932. El acta número 2, de diez de octubre de 1932, recoge en el punto dos: "realizar algunas excursiones escolares para dar al aire libre lecciones de cosas de la naturaleza, buscando al mismo tiempo el ejercicio físico de las niñas y el conocimiento e intercambio de ideas con las maestras y niñas de los pueblos limítrofes". Asimismo el acta número 17, de 3 de enero de 1934, trata en su punto tercero: "Aprovechando la tarde de un jueves, se harán paseos escolares, una vez al mes, los tres grados, cada uno a diferente sitio. El fin de estas excursiones será principalmente educativo y se dedicarán a la enseñanza de la Geografía (sic) y Ciencias Naturales. Cada maestra llevará un Registro de paseos escolares de cada grado. Al siguiente día de efectuado el paseo las niñas de Segundo y Tercer grado, harán un ejercicio de redacción de lo aprendido o visto en él".

21 CEMUPE. FDoc. $(92,98)$. Caja 5. Alumna $M^{a}$ de las Mercedes Crespo Asensio.

22 CEMUPE. FDoc. Cuaderno de la alumna Rufina Miguel Manzano. Curso escolar 1935-36. Escuela nacional de El Perdigón (Zamora)

23 CEMUPE. FDoc. $(95,96)$. Caja 5. Alumna $M^{a}$ de los Ángeles Asla Polo. (CMPM). Prác- 
La tarde de los jueves se dedica a actividades de periodismo, expansión cultural, orden y museo escolar, cultivos e industrias domésticas, canto y deporte. ${ }^{24}$

Por tanto, en este periodo republicano, las teorías de la Escuela Nueva, del socialismo y de la ILE, que ya habían venido siendo introducidas en el primer tercio del siglo XX poco a poco en escuelas regentadas por maestros/as reformadores y progresistas, se consolidan, generalizan y afianzan. La llegada de la Segunda República supone la proyección de los ideales que se sustentaban en el plano teórico al plano de la praxis. El niño como centro de su propio proceso de aprendizaje, el activismo, los centros de interés de Decroly, la escuela para la vida, el interés por conocer, el periódico escolar de Freinet, la enseñanza intuitiva y el maestro como guía tienen en la escuela el espacio preciso para su aplicación.

\section{Otras consideraciones de la praxis escolar}

Las fuentes consultadas muestran las distintas iniciativas innovadoras que la República impulsa en el campo de la enseñanza primaria. Si bien no en su totalidad, sí gran parte de ellas, ya que estos documentos hacen referencia a la política educativa relacionada con el concepto de escuela, muy amplio en extensión, que los responsables del Ministerio de Instrucción Pública esgrimían. Así, en las escrituras de maestros y alumnos se encuentran alusiones directas- en otro momento se iniciará su estudio- a la utilización de cánticos escolares: "con el fin de armonizar un poco más la enseñanza, durante las horas de labor les enseñé algunos himnos patrióticos" 25 ; la presencia de los primeros ejercicios de gimnasia; la preocupación por las condiciones higiénicas del localescuela, su ubicación, emplazamiento, iluminación, capacidad de superficie y volumen, orientación, ventilación del mismo y dependencias; el régimen interior que incluye la descripción de la matrícula, el horario, la rotación, el recreo, las enseñanzas, el aumento de docentes y la existencia de material.

ticas realizadas en la escuela unitaria de niñas de Lugo.1934-35

24 CEMUPE. FDoc. (94,97). Caja 5. Alumna Asunción Alonso Tajadura. (CMPM). Prácticas realizadas en la escuela graduada aneja a la Normal de Valladolid.1935

25 CEMUPE. FDoc. (50). Caja 5. Alumna Pascuala del Brío Casaseca. (CMPM). Escuela nacional de El Piñero (Zamora). 1934. 
La cultura escolar de la Segunda República española. Legislación, teoría y praxis escolar

Bienvenido Martín Fraile, Isabel Ramos Ruiz y Pablo Álvarez Domínguez

Entre las innovaciones se han introducido carpetas individuales para cada uno de los niños, cuadernos de trabajo en los que diariamente hacen sus ejercicios, enciclopedias escolares, prácticas de agricultura, gimnasia escolar, iniciación al juego del futbol, paseos escolares, lecciones de cosas, trabajos manuales, registro paidológico. ${ }^{26}$

Asimismo remarcar la presencia de instituciones complementarias: mutualidad escolar, museo, biblioteca, roperos, cantinas escolares, clases de adultos, gabinetes antropométricos y asociación de amigos de la escuela que intenta hacer partícipes a los padres de la obra escolar.

También aparece un tema recurrente entre las propuestas ministeriales como fue la coeducación, un apartado que no llegó a implantarse en un grado total en esta zona geográfica estudiada. Los cuadernos de Memorias de Prácticas de los alumnos de Magisterio, los de preparación de lecciones de los maestros y los cuadernos de alumnos muestran una implantación desigual, y en muchos casos se presenta una escuela primaria con separación de sexos, excluyendo solamente a la escuela primaria unitaria, donde la coeducación se lleva a cabo por falta de medios personales, al estar la escuela bajo la dirección de un único maestro o maestra, o de espacios, al contar con un único local para impartir la enseñanza.

\section{Reflexión final}

El trabajo se ha centrado en el estudio de la escuela primaria en la Segunda República en zonas rurales donde vive la mayoría de la población con un alto porcentaje de analfabetismo y unas condiciones deplorables para el desarrollo de la enseñanza. Por tanto, se ha circunscrito a un ámbito geográfico específico, de donde proceden las fuentes documentales, para evitar una excesiva generalización. Se ha analizado un área centrada en el noroeste de España y cercana a Portugal, con unas condiciones sociales, económicas y educativas propias. No obstante, sería deseable que poco a poco se realizaran investigaciones en distintas áreas territoriales que abordasen la misma temática y así obtener una visión de conjunto a nivel nacional.

26 CEMUPE. FDoc. (129) Caja 2. Alumno Felipe Hernández Garzón. Escuela de Villardondiego (Zamora). 1932. En su memoria de prácticas deja constancia del cambio sufrido en la escuela y que él ha observado durante su estancia práctica en lo referente a la llegada de material. 
En el estudio se ha observado que los principios de la Segunda República para la escuela se cumplen en el área geográfica analizada. Una escuela en donde se sitúa el foco de atención para mejorarla, teniendo como principio que el progreso de un país se encuentra vinculado a su cultura y educación. No se escatiman esfuerzos tanto en la formación y dignificación del Magisterio como en la propuesta de un modelo educativo reformista e innovador. Una evidencia que se recoge en diferentes documentos de carácter legislativo, en las teorías afines a la ILE, al socialismo y a la Escuela Nueva y en su aplicación al ámbito escolar. La evidencia de este camino que recorren conjuntamente los ámbitos normativo, teórico y práctico se ha ido analizando en distintas fuentes: cuadernos de alumnos, de Memorias de Prácticas de Magisterio y Libros de Actas principalmente.

El análisis de las Memorias de Prácticas, principalmente de zonas rurales, y en número menor aquellas de escuelas graduadas de ciudades, señalan el pensar y el sentir de cada escuela en el testimonio recogido por la pluma del alumno que expresa cuanto ve y observa desde el momento de entrada en la escuela hasta el final de sus prácticas. Se escenifica la distribución de alumnos, espacios y anota las pautas metodológicas y actividades académicas desarrollada por cada maestro a través de sus horas de clase y patios.

El análisis de los cuadernos de los alumnos favorece una visión más completa de ésta. Informa de las materias dadas, la importancia concedida a cada una, la metodología usada, la vida diaria, la relación entre sus protagonistas o los valores transmitidos. En suma, fuentes y documentos proporcionan una información compleja de la que se extrae consideraciones significativas. En primer lugar, que la escuela graduada tardó en implantarse, la mayoría de las escuelas eran unitarias diferenciándose de los centros escolares del mundo urbano donde sí empezaba a extenderse la graduación. En segundo lugar, Ilama la atención el impulso de una metodología activa y participativa por parte de los maestros que busca la implicación del alumno en su propio aprendizaje. En tercer lugar, el tema de la coeducación queda claro al contemplar que no hubo un currículum único, sino diferenciado por sexos y que no se produjo una implantación radical, sino que se iba produciendo lentamente. El laicismo en la escuela, en cambio, fue una realidad a lo largo de todo el periodo. También se ha consignado que la enseñanza de principios democráticos y de ciudadanía se plasmó en los ejercicios diarios del aula con el fin de 
La cultura escolar de la Segunda República española. Legislación, teoría y praxis escolar

Bienvenido Martín Fraile, Isabel Ramos Ruiz y Pablo Álvarez Domínguez

conseguir un modelo de hombre y mujer iguales, respetuosos, participativos y solidarios.

Aun siendo conscientes de la dificultad de implantación de las reformas y cambios educativos en el tiempo y de la resistencia a los cambios de prácticas consolidadas en el ámbito escolar, es posible visualizar lo que se pretende en este trabajo. Las fuentes consultadas evidencian la plasmación en la escuela de las disposiciones legales con su bagaje normativo y teórico, revelando una conexión real y eficiente entre los tres ámbitos que caracterizan la cultura escolar en este periodo republicano.

Se desconoce con exactitud el número de maestros que apoyaron el proyecto; se trata de personas donde unas se comprometieron y otras no, siendo más resistentes al cambio, por lo que se necesita hacer una revisión histórica de forma objetiva y científica que permita conocer los educadores y pedagogos participantes y su grado de implicación. Sería aconsejable, como se ha comentado anteriormente, continuar con estas investigaciones que aborden áreas geográficas concretas que proporcionen, de cara al primer centenario de la Segunda República, ya próximo, la evidencia del traslado a la praxis escolar de los planteamientos ideológicos y principios teóricos educativos, dando lugar a una visión real, evitando generalizaciones en base a focalizaciones locales realizadas en algunos casos sin determinar el grado real de implantación

\section{Referencias}

Barceló, G., Comás, F. y Sureda, B. (2016). Abriendo la caja negra: la escuela pública española de posguerra. Revista de Educación, 371, 61-82.

Beas, M. y González, E. (2016). Estudio de las emociones en las consignas de cuadernos españoles. Curso 1964-1965. Revista de Estudios Sociales de la Universidad de los Andes, 58, 52-62

Barreiro, H. (1983).Repercusiones de la revolución ideológica y científica del último tercio del siglo XIX en las innovaciones educativas de la Institución Libre de Enseñanza. Revista de Historia de la Educación. Revista Interuniversitaria, 2, 209-215.

Capitán, A. (1986). Historia del pensamiento pedagógico en Europa. Madrid: Dykinson. Vol. II.

Cossío, M.B. (1929). De su jornada. Madrid: Imprenta de Blas.

Escolano, A. (2006). La cultura de la escuela en el sistema educativo liberal. En A. Escolano. (Dir.). Historia ilustrada de la escuela en España. Dos siglos de perspectiva histórica. (pp. 23-46). Madrid: Fundación Germán Sánchez Ruipérez. (2007). La cultura material de la escuela. En A. Escolano (ed.). La cultura mate- 
La cultura escolar de la Segunda República española. Legislación, teoría y praxis escolar

Bienvenido Martín Fraile, Isabel Ramos Ruiz y Pablo Álvarez Domínguez

rial de la escuela. En el centenario de la JAE,(1907-2007). (pp.15-27). Berlanga de Duero: CEINCE.

Juliá, D. (1995). La cultura scolaire comme objet historique. The colonial experience in education. Paedagogica Historica Supl. Series, 1, 353-382.

Llopis, R. (1927). Hacia una escuela más humana. BILE, marzo.

Martín, B. (2015). La cultura escolar y el oficio de maestro. Educación XXI. UNED, 18 (1), pp. 147-166.

Martín, B. y Ramos, I. (2010). Segunda República y escuela. Valores sociales y cívicos en los cuadernos de rotación. En E. Collellldemont (coord.). Memoria, ciudadanía y museos de educación. (pp. 171-181). IV Jornadas de la SEPHE. Vic: MUVIP.

(2014). Una nueva manera de interpretar los cuadernos escolares: Las escrituras al margen. History of Education \& Children Literature, Macerata, IX, (2), pp. 643-66 (2015). La historia contada en los cuadernos escolares. Madrid: Catarata.

Molero, A. (2010). La inspección educativa y el perfeccionamiento del profesorado: una visión retrospectiva. Participación Educativa, 13, 170-179.

Pozo, Ma M. del y Rabazas,T. (2013). Políticas educativas y prácticas escolares: la aplicación de la Ley de Enseñanza primaria de 1945 en las aulas. Bordón, 65, 119-133.

Revista de Pedagogía (1922-1936). Madrid: La Revista

Sustar, B. (2011). What was school like? Testimonies on schooling as part of collector, exhibition, research and publicist activity of the museum. En AM. Augschöll, (coord.). XIV International Symposium for School Life and School History Collections, Freue Universität Bozen, Brixen.

Viñao, A. (1996). Por una historia de la cultura escrita: observaciones y reflexiones. Signo. Revista de Historia de la Cultura Escrita, 3, 41-68.

\section{Relación de fuentes documentales}

CEMUPE. Centro Museo Pedagógico de la Universidad de Salamanca. Fondo documental de cuadernos escolares.

Constitución Española de 1931

Decreto de 4 de mayo de 1931. Colección Legislativa de España, T. CXXIV

Circular de 12 de enero de 1932, firmada por el Director General de Primera Enseñanza, Rodolfo Llopis.

Circular de 28 de marzo de 1936, firmada por el Ministro de Instrucción Pública, Marcelino Domingo. 
